\title{
Supersonic swirling characteristics of natural gas in convergent-divergent nozzles
}

\author{
Wen Chuang, Cao Xuewen*, Yang Yan and Zhang Jing \\ Department of Oil and Gas Engineering, China University of Petroleum, Qingdao, Shandong 266555, China \\ (C) China University of Petroleum (Beijing) and Springer-Verlag Berlin Heidelberg 2011
}

\begin{abstract}
The supersonic nozzle is a new apparatus which can be used to condense and separate water and heavy hydrocarbons from natural gas. The swirling separation of natural gas in the convergentdivergent nozzle was numerically simulated based on a new design which incorporates a central body. Axial distribution of the main parameters of gas flow was investigated, while the basic parameters of gas flow were obtained as functions of radius at the nozzle exit. The effect of the nozzle geometry on the swirling separation was analyzed. The numerical results show that water and heavy hydrocarbons can be condensed and separated from natural gas under the combined effect of the low temperature ( -80 ${ }^{\circ} \mathrm{C}$ ) and the centrifugal field $(482,400 \mathrm{~g}, \mathrm{~g}$ is the acceleration of gravity). The gas dynamic parameters are uniformly distributed correspondingly in the radial central region of the channel, for example the distribution range of the static temperature and the centrifugal acceleration are from -80 to $-55{ }^{\circ} \mathrm{C}$ and $220,000 \mathrm{~g}$ to $500,000 \mathrm{~g}$, respectively, which would create good conditions for the cyclone separation of the liquids. However, high gradients of gas dynamic parameters near the channel walls may impair the process of separation. The geometry of the nozzle has a great influence on the separation performance. Increasing the nozzle convergent angle can improve the separation efficiency. The swirling natural gas can be well separated when the divergent angle takes values from $4^{\circ}$ to $12^{\circ}$ in the convergent-divergent nozzle.
\end{abstract}

Key words: Swirling flow, convergent-divergent nozzle, supersonic, natural gas separation, numerical calculation

\section{Introduction}

New technologies have to be developed to efficiently meet the offshore requirements due to the discovery of offshore reservoirs. Supersonic nozzles have been introduced to treat natural gas for offshore applications (Okimoto and Brouwer, 2002; Betting et al, 2003; Alferov et al, 2004; Alfyorov et al, 2005; Liu et al, 2005). In a convergent-divergent nozzle, the swirling flow, generated by the vanes at the entrance of the nozzle, expands to supersonic velocities, resulting in lower temperatures. Nucleation of water and heavy hydrocarbons occurs, followed by growth of liquid droplets (Brouwer and Epsom, 2003; Betting and Epsom, 2007; Jiang et al, 2008a; 2008b; Wen et al, 2010). The swirl strength increases strongly because of the contraction in the nozzle. Therefore, the liquid droplets are centrifuged onto the walls, creating a liquid film, and are then separated. The swirling flow through the convergent-divergent nozzle determines the separation efficiency of natural gas. The mixing flow field of nitrogen and water vapor was numerically calculated in an axial flow supersonic nozzle (Jiang et al, 2008a; 2008b). The natural

*Corresponding author. email: caoxw@upc.edu.cn

Received June 11, 2010 gas behavior was illustrated when it was considered to be real and the calculated properties might become inaccurate when the gas was assumed to be ideal in axial flow supersonic nozzles (Jassim et al, 2008a; 2008b). The effect of nozzle geometry was discussed by inserting a constant area channel between the convergent and divergent parts of the nozzle (Jassim et al, 2008a; 2008b). Design methods of axial flow supersonic nozzles for natural gas separation are numerically calculated, and the flow field was obtained (Cao et al, 2007). A computational model linked to MATLAB and HYSYS package was presented to predict the effect of different parameters such as the inlet pressure, inlet temperature, and flow rate on the behavior of the working fluid (Karimi and Abdi, 2009).

A great deal of recent scientific research on the performance of the convergent-divergent nozzle has mainly focused on the axial flow nozzle, in which the upstream inflow is steady, and only a few investigations have been performed on the swirling flow. As a result, it is necessary to investigate the swirling flow in a convergent-divergent nozzle for natural gas separation.

In this paper, a new annular convergent-divergent nozzle is designed, which incorporates a central body. The axial and radial distribution of the main parameters of gas flow and the effect of the nozzle geometry are analyzed and discussed. 


\section{Flow behavior in convergent-divergent nozzles}

The adiabatic cooling is accomplished through a convergent-divergent nozzle, which achieves an isentropic expansion efficiency of more than $80 \%$. The temperature of flow is lowered based on the principle of gas expansion where no refrigerant is needed. The gas speed in this device is so high that it results in a maximum gas residence time less than 2 milliseconds and prevents formation of gas hydrate.

This new annular nozzle incorporates a central body, which allows the principle of conservation of angular momentum to be harnessed. The channel, between the wall and the central body, forms a convergent-divergent nozzle, which is composed of three sections: the convergent (subsonic zone), throat (critical zone), and divergent sections (supersonic zone), as shown in Fig. 1.

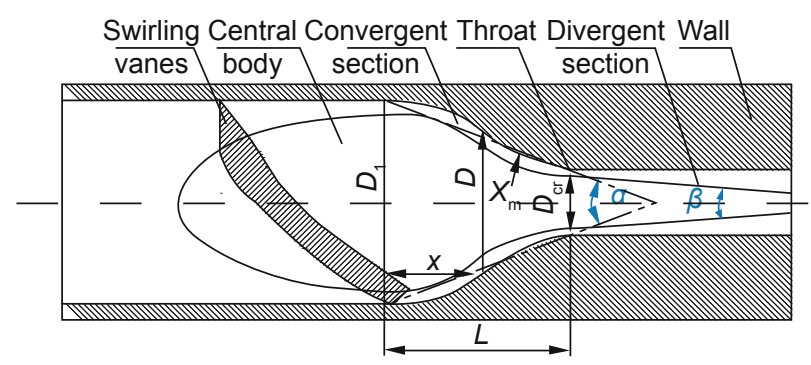

Fig. 1 The structure of the nozzle

Within the convergent section, the flow is accelerated so that the speed of sound is reached at the throat zone. In order to obtain the sonic velocity at the throat, it is necessary to keep that the inlet diameter is larger than $\sqrt{5}$ times the throat diameter (Man et al, 1997). The convergent length is typically equal to or greater than the throat diameter. In this research, the dimensions of the convergent section of the nozzle are calculated by Eq. (1).

$$
\begin{cases}\frac{D-D_{\text {cr }}}{D_{1}-D_{\text {cr }}}=1-\frac{1}{X_{\mathrm{m}}{ }^{2}}\left(\frac{x}{L}\right)^{3} & \left(\frac{x}{L} \leq X_{\mathrm{m}}\right) \\ \frac{D-D_{\text {cr }}}{D_{1}-D_{\text {cr }}}=\frac{1}{\left(1-X_{\mathrm{m}}\right)^{2}}\left(1-\frac{x}{L}\right)^{3} & \left(\frac{x}{L}>X_{\mathrm{m}}\right)\end{cases}
$$

where $D_{1}$ is the inlet diameter, $\mathrm{m} ; D_{\text {cr }}$ is the throat diameter, $\mathrm{m} ; L$ is the convergent length, $\mathrm{m} ; X_{\mathrm{m}}$ is the relative coordinate of this convergent curve; $x$ is the distance between arbitrary cross section and the inlet, $\mathrm{m}$; and $D$ is the convergent diameter at an arbitrary cross section of $x, \mathrm{~m}$, as shown in Fig. 1.

After the gas reaches the throat, the divergent section of the nozzle can further accelerate the flow to supersonic velocity and the temperature of flow is lowered, which leads to the nucleation of water and heavy hydrocarbons, followed by growth of liquid droplets. However, under certain conditions, it is likely that the flow cannot expand isentropically to the exit pressure; hence, a shockwave occurs. As a result of the gradients in temperature and velocity, which are created by the shockwaves, heat is transferred and energy is dissipated within the gas. Therefore, it is necessary to prevent the occurrence of shockwaves in the divergent channel of the nozzle for this apparatus.

An important aspect of the numerical computation is to swirl the natural gas flow. The swirling device consists of 12 vanes turned through $45^{\circ}$ and located in the subsonic section of the channel on the central body, as shown in Fig. 1. The central line of the vane was an arc. The chord length and twist angle of the vane are $60 \mathrm{~mm}$ and $30^{\circ}$, respectively. This fact must be taken into account in further numerical calculation.

\section{Mathematical model}

\subsection{Governing equations}

In the convergent-divergent nozzle, the natural gas is compressible and forms a strong swirling flow. The fluid flow characteristics in the nozzle can be depicted by the partial differential equations including mass equation (continuity equation), momentum equation, and energy equation, which are the basis for the calculation and simulation, described as Eqs. (2)-(4).

$$
\begin{aligned}
& \frac{\partial \rho}{\partial t}+\frac{\partial}{\partial x_{i}}\left(\rho u_{i}\right)=0 \\
& \frac{\partial}{\partial t}\left(\rho u_{i}\right)+\frac{\partial}{\partial x_{j}}\left(\rho u_{i} u_{j}\right)+\frac{\partial p}{\partial x_{i}}-\frac{\partial \tau_{i j}}{\partial x_{j}}-\frac{\partial \tau_{i j}^{-1}}{\partial x_{j}}=0 \\
& \frac{\partial}{\partial t}(\rho E)+\frac{\partial}{\partial x_{j}}\left(\rho u_{j} E+u_{j} p\right)-\frac{\partial}{\partial x_{j}}\left(u_{i} \tau_{i j}+u_{i} \tau_{i j}^{-1}\right) \\
& +\frac{\partial}{\partial x_{j}}\left(q_{j}+q_{j}^{-1}\right)=0
\end{aligned}
$$

where $\rho, u$, and $p$ are the gas density $\left(\mathrm{kg} / \mathrm{m}^{3}\right)$, velocity $(\mathrm{m} / \mathrm{s})$, and pressure $(\mathrm{Pa})$, respectively. $\tau_{i j}$ is the viscous stress, $\mathrm{N} / \mathrm{m}^{2}$; $E$ is the total energy, $\mathrm{J} / \mathrm{kg} ; q_{j}$ is the heat flux, $\mathrm{W} / \mathrm{m}^{2} ; t$ is the time, s.

\subsection{Turbulence model and solution algorithm}

Because the values of the Reynolds number in the nozzle are very high, the modeling of the Reynolds stress plays a crucial role in the flow prediction. A turbulence model is a means of deriving the Reynolds stresses in order to close the mean flow equations. The RNG $k-\varepsilon$ turbulence model has an additional term in its $\varepsilon$ equation to consider the effect of swirl on turbulence, enhancing the accuracy for swirling flows. That is, the RNG $k-\varepsilon$ model provides an option to account for the effect of swirl or rotation by modifying the turbulent viscosity appropriately (Pope, 2000). Therefore, the RNG $k-\varepsilon$ model is used here for the computational calculation.

In the numerical calculation, the finite volume method and the second-order upwind scheme were used; and the wall function was introduced to model the flow near the wall, while the SIMPLE algorithm (Patankar and Spalding, 1972; Patankar, 1980) was applied to couple the velocity field and pressure. 


\subsection{Mesh and boundary conditions}

The quality of the mesh plays an important role in the accuracy and stability of the numerical calculation. The polyhedral mesh allows the flexibility of an unstructured mesh to be applied to a complex geometry. Therefore, the nozzle is meshed by the unstructured tetrahedral grids, as a result of the twisted swirling vanes, as shown in Fig. 2.

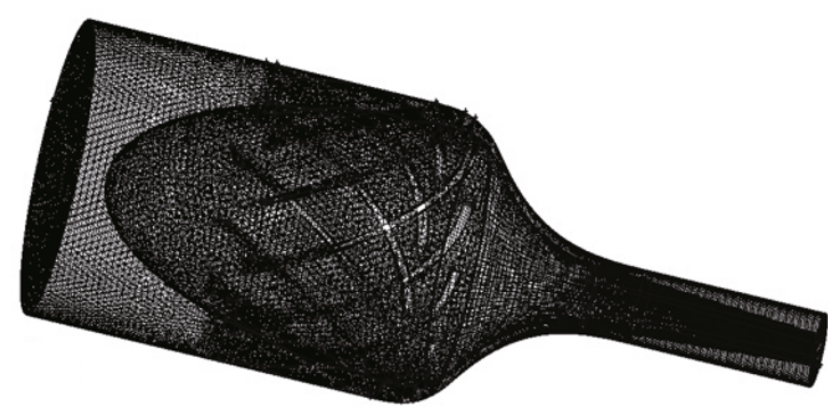

Fig. 2 Mesh topology of the geometry

According to the flow characteristics of the supersonic compressible fluid, the boundary conditions are imposed as follows: pressure boundary conditions for the inlet and outlet of the nozzle, respectively and the no-slip and adiabatic boundary conditions are specified for the walls.

Parameters of pressure boundary conditions for the inlet and outlet are presented as follows.

Pressure inlet: total pressure (absolute pressure) and total temperature at the nozzle inlet are set to be $4 \mathrm{MPa}$ and $303 \mathrm{~K}$, respectively. Turbulence intensity and viscosity ratio are assigned as turbulence parameters. Turbulence intensity and viscosity ratio are 0.048 and 1 , respectively, for the calculation.

Pressure outlet: the static pressure is not a necessary condition for the reason that the velocity of the gas flow is supersonic at the nozzle outlet. The stagnation temperature is $290 \mathrm{~K}$. Turbulence intensity and viscosity ratio are 0.025 and 10 , respectively.

\subsection{Equation of state and convergence conditions}

In this paper, it is assumed that the gas obeys the model equation of state which enables one to take into account the effect of super-compressibility in Eq. (5).

$$
p=z \rho R T / M
$$

where $p, \rho, T, M, R$, and $z$ are the gas pressure $(\mathrm{Pa})$, density $\left(\mathrm{kg} / \mathrm{m}^{3}\right)$, temperature $(\mathrm{K})$, molar mass $(\mathrm{kg} / \mathrm{mol})$, gas constant $(\mathrm{J} /(\mathrm{K} \cdot \mathrm{mol}))$, and coefficient of super-compressibility (dimensionless), respectively. The coefficient of supercompressibility $z$ is a function of pressure and temperature.

The mole composition of natural gas taken from the Baimiao Field is as follows: $2.04 \% \mathrm{~N}_{2}, 0.45 \% \mathrm{CO}_{2}, 0.03 \%$ $\mathrm{H}_{2} \mathrm{O}, 91.36 \% \mathrm{CH}_{4}, 3.63 \% \mathrm{C}_{2} \mathrm{H}_{6}, 1.44 \% \mathrm{C}_{3} \mathrm{H}_{8}, 0.26 \% i-\mathrm{C}_{4} \mathrm{H}_{10}$, $0.46 \% n-\mathrm{C}_{4} \mathrm{H}_{10}, 0.17 \% i-\mathrm{C}_{5} \mathrm{H}_{12}$, and $0.16 \% n-\mathrm{C}_{5} \mathrm{H}_{12}$.

In this simulation, the convergence criterion is $10^{-6}$ for the energy equation and $10^{-3}$ for all other equations. The total number and time of the iterations is about 2,300 and $2 \mathrm{~h}$, respectively. The computational platform employs a $\mathrm{PC}$ with a Pentium 2.8 G Duo CPU, 4 G RAM-memory and 320 GB hard disc memory.

\subsection{Verification and comparison}

\subsubsection{Verification of grid independence}

The mesh density affects the numerical results, but the results should be stable when the mesh size is in a certain range. In this study, the grid independence was investigated with the mesh number of 233,952 and 1008,894, respectively. The Mach number ( $M a$ ) of natural gas in the nozzle is shown in Figs. 3 and 4. Mach number is defined as the speed ratio, referenced to the speed of sound.

$$
M a=v_{\mathrm{g}} / a
$$

where $M a, v_{\mathrm{g}}$, and $a$ are the Mach number (dimensionless), the gas speed $(\mathrm{m} / \mathrm{s})$, and the speed of sound $(\mathrm{m} / \mathrm{s})$, respectively.

The axial distributions of the Mach number are consistent. So grid dependency studies have shown that larger grids do not necessarily influence the accuracy of the solution in the case of the supersonic nozzle flow, while the mesh size is in a certain range. Therefore, we generated the nozzle mesh in the following simulation from the economic perspective of the calculation.

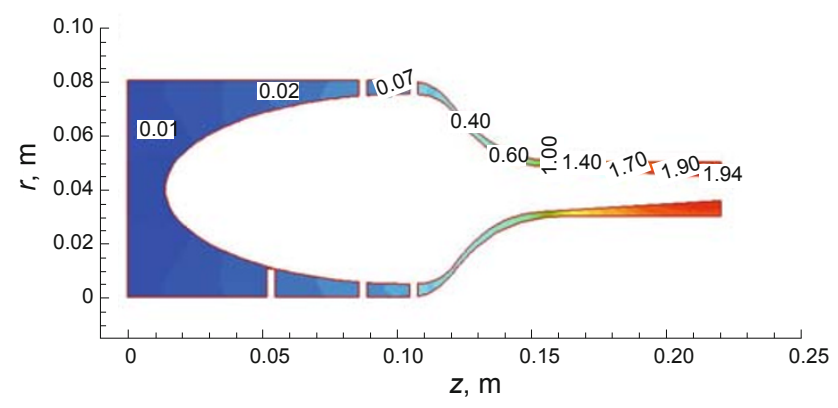

Fig. 3 Numerical result of 233952 grids: Mach number

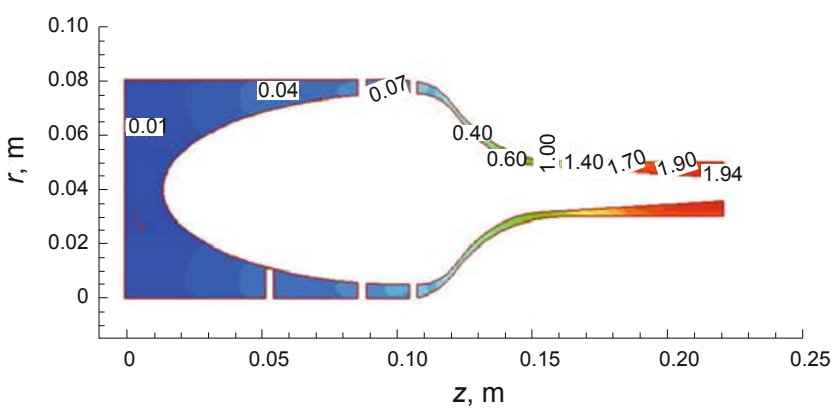

Fig. 4 Numerical result of 1008894 grids: Mach number

\subsubsection{Model validation}

Although the numerical solution methods were discussed detailed in the above paragraphs, it is necessary to validate whether these methods could be used to calculate the complex swirling flow in a convergent-divergent nozzle. This presentation will compare the numerical results with analytical and experimental data, picked from the research of Boerner et al (1972). Fig. 5 shows the dimensionless 
parameter $M$, which is the ratio of the mass flow rate in the presence of swirl to the mass flow rate for a corresponding non-swirling flow, as a function of swirl parameter $S$, which is the ratio of the tangential velocity to the critical velocity at the throat. The mass flow rate decreases with an increase in the swirl strength. The numerical results are in agreement with the analytical and experimental data.

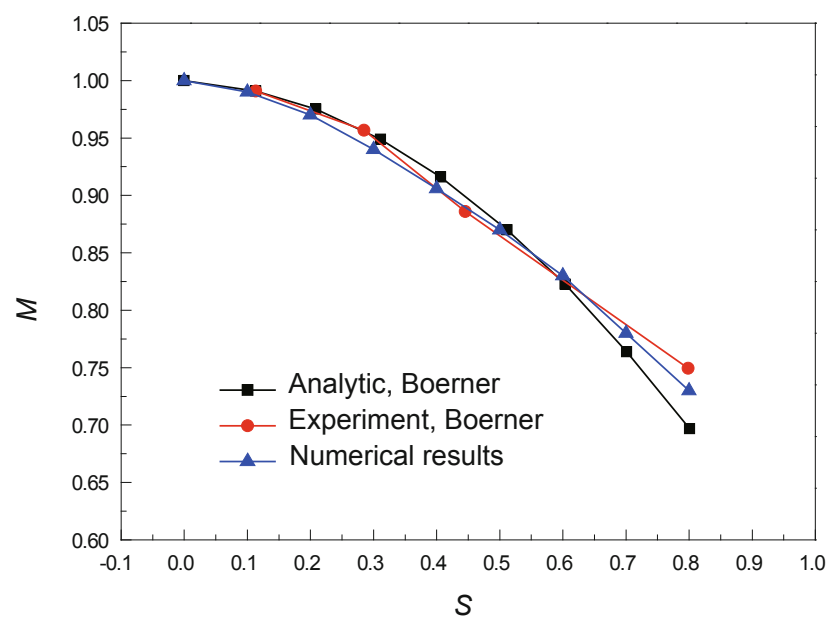

Fig. 5 Effects of swirl strength on the mass flow rate

\section{Results and discussion}

The flow field was numerically simulated in the convergent-divergent nozzle, based on the new design mentioned above and the numerical methods. The axial and radial distribution of the gas dynamics parameters and the effects of the nozzle geometry were obtained. The pressure, flow rate, and temperature of natural gas for the design of the convergent-divergent nozzle are $4 \mathrm{MPa}, 10 \times 10^{4} \mathrm{Nm}^{3} / \mathrm{d}$, and $30{ }^{\circ} \mathrm{C}$, respectively. The nozzle is about $220 \mathrm{~mm}$ long and $80 \mathrm{~mm}$ in the inlet diameter. The diameters of the central body and the wall at the throat are 13.78 and $19.32 \mathrm{~mm}$, respectively; and the diameters of the central body and the wall at the nozzle outlet are 7.12 and $19.32 \mathrm{~mm}$, respectively.

\subsection{Axial distribution of the gas dynamics parameters}

Fig. 6 depicts the axial distributions of the average static temperature and tangential velocity at the cross section. Gas expands in the convergent-divergent nozzle to supersonic velocities, resulting in lower temperatures $\left(-80^{\circ} \mathrm{C}\right)$. The static temperature decreases slowly in the convergent section of the nozzle, while gas expansion induces a rapid decrease in the divergent part. When the static temperature of natural gas becomes lower than the condensation temperature of some component, and if there is enough time for condensation, the nucleation will occur followed by growth of liquid droplets. The swirling motion is generated by the vanes at the entrance of the nozzle. The swirl strength increases strongly, due to the contraction in the annular nozzle and the second convergence of the central body, resulting in a centrifugal field of about $482,400 \mathrm{~g}$ ( $\mathrm{g}$ is the acceleration of gravity). The liquid droplets are centrifuged onto the walls, creating a liquid film, owing to the centrifugal acceleration field. The central body

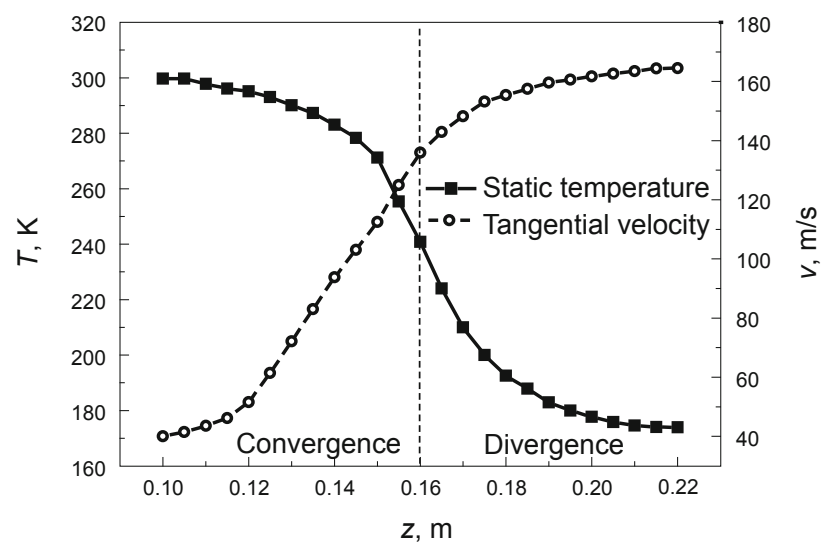

Fig. 6 Average static temperature and tangential velocity of gas flow in the nozzle

ensures that the vortex is concentric, allowing the separation efficiency to be significantly improved.

\subsection{Radial distribution of the gas dynamics parameters}

As result of the swirling flow generated by the twisted vanes, the main parameters of gas flow (velocity components, temperature, etc) are non-uniformly distributed radially in the new designed nozzle. The radial distribution of gas velocity involves a redistribution of static pressure in the channel, which leads to the emergence of return flows. In the nozzle divergent part the vortex causes centrifugation of the droplets.

The presence of the strong swirling flow in the nozzle causes the nonuniform radial distribution of the main parameters of gas flow. Therefore, it is necessary to evaluate the effect of the radial distribution of the main parameters of gas flow on the process of separation. The distribution of the gas temperature and the tangential velocity at the nozzle exit are given in Figs. 7-8.

The vortexes are not dissipated in the nozzle due to the presence of the central body. On the contrary, the swirl strength increases strongly because of the contraction of the central body, which results in the non-uniform radial distribution of the main parameters of gas flow. However, in the radial central region of the channel, the main parameters are uniformly distributed. The tangential velocity is radially distributed from 100 to $160 \mathrm{~m} / \mathrm{s}$, on the radius from 44.5 to $48.5 \mathrm{~mm}$, and the static temperature is radially distributed from 193 to $218 \mathrm{~K}$. In other words, the centrifugal acceleration is up to $220,000 \mathrm{~g}$ to $500,000 \mathrm{~g}$, as well as the static temperature takes values from -80 to $-55{ }^{\circ} \mathrm{C}$, both of which would create good conditions for the cyclone separation of the liquids. On the other hand, it can be seen that there are high gradients of gas dynamic parameters in the vicinity of the channel walls. The presence of positive gradients of the velocity on the channel walls may lead to vortex stirring of the boundary layer containing condensed droplets. This may cause undesirable mixing of the already condensed gas components with the main flow. The high temperature gradient near the walls can cause higher evaporability of the condensed gas components. Moreover, it is possible to impair the process of separation. Therefore, it 


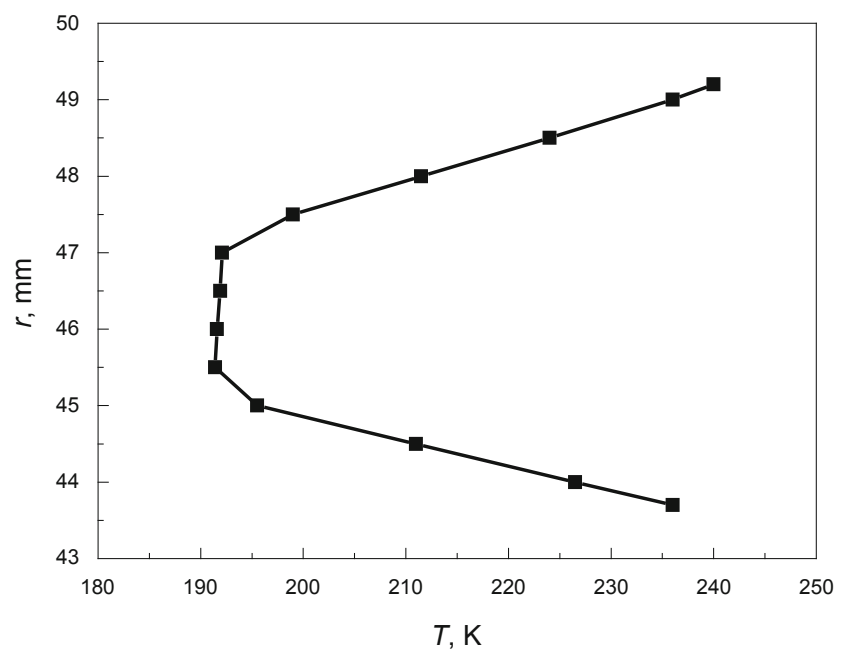

Fig. 7 Radial distribution of the static temperature at the nozzle exit

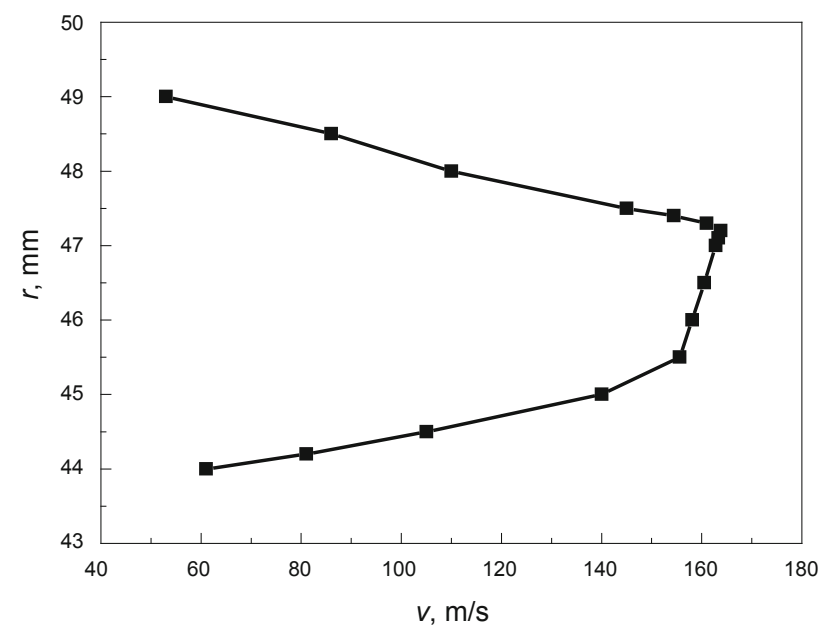

Fig. 8 Radial distribution of the tangential velocity at the nozzle exit

is demonstrated that the emergence of the non-uniformity of radial distribution of the gas dynamic parameters significantly affects the gas/liquid separation.

\subsection{Effect of the nozzle geometry}

\subsubsection{Effect of the convergent angle}

The numerical computation of the effect of the convergent angle on the swirling separation in the nozzle was carried out, under the assumptions that the divergent angle is constant. The angle $\alpha$ between the two contraction curves is defined to be the convergent angle, as shown in Fig. 1. The values of convergent angle are $20^{\circ}, 30^{\circ}, 40^{\circ}, 50^{\circ}, 60^{\circ}, 70^{\circ}, 80^{\circ}, 90^{\circ}$, $100^{\circ}, 110^{\circ}$, and $120^{\circ}$, respectively. The numerical results are shown in Fig. 9. It is obvious that the convergent angle has a significant influence on the average static temperature and the tangential velocity at the outlet when it is less than $60^{\circ}$, but after that the influence is insignificant. Although the average static temperature at the exit increases $4.3{ }^{\circ} \mathrm{C}$ when the convergent angle increases from $20^{\circ}$ to $60^{\circ}$, the centrifugal acceleration at the outlet increases sharply, from 147,000 $\mathrm{g}$ to $380,000 \mathrm{~g}$. In this case, the average centrifugal acceleration has a greater influence on the swirling separation of natural gas than the average static temperature.

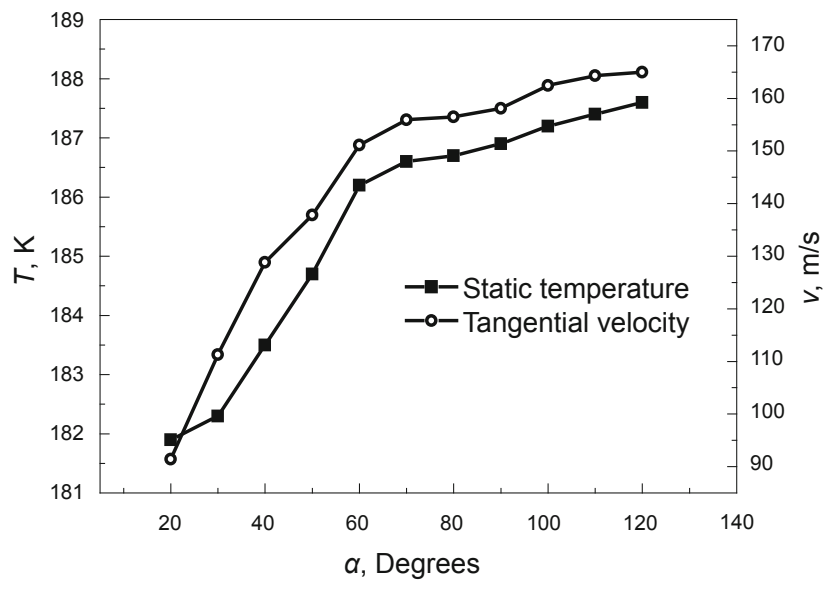

Fig. 9 Effects of convergent angle

\subsubsection{Effect of the divergent angle}

The influences of the divergent angle on the swirling separation of natural gas in the convergent-divergent nozzle were numerically simulated, under the conditions that the convergent angle is constant. The angle $\beta$ between the two divergent curves is defined to be the divergent angle, as Fig. 1 shows. The results of the simulation are shown in Fig. 10.

The average static temperature at the nozzle exit is high when the divergent angle is less than $4^{\circ}$. The reasons are that the boundary layers are the main influencing factors on the performance of the nozzle when the divergent angle is small. In this case, the distribution of the boundary layer thickness along the walls of the channel, influences the actual flow in the nozzle and decreases the effective flow area. Therefore, the nozzle cannot accelerate the flow to the expected supersonic velocity, which leads to an increase in the static temperature.

When the divergent angle is $4^{\circ}-12^{\circ}$, the influence of the boundary layer is insignificant and the average static temperature is kept between -80.3 and $-76.6{ }^{\circ} \mathrm{C}$. Meanwhile, the average centrifugal acceleration takes values from $300,000 \mathrm{~g}$ to $330,000 \mathrm{~g}$. Therefore, better separation performance of the convergent-divergent nozzle for natural gas, is achieved.

Extending the divergent angle consecutively, the boundary layer starts to breaking away from the walls of the channel,

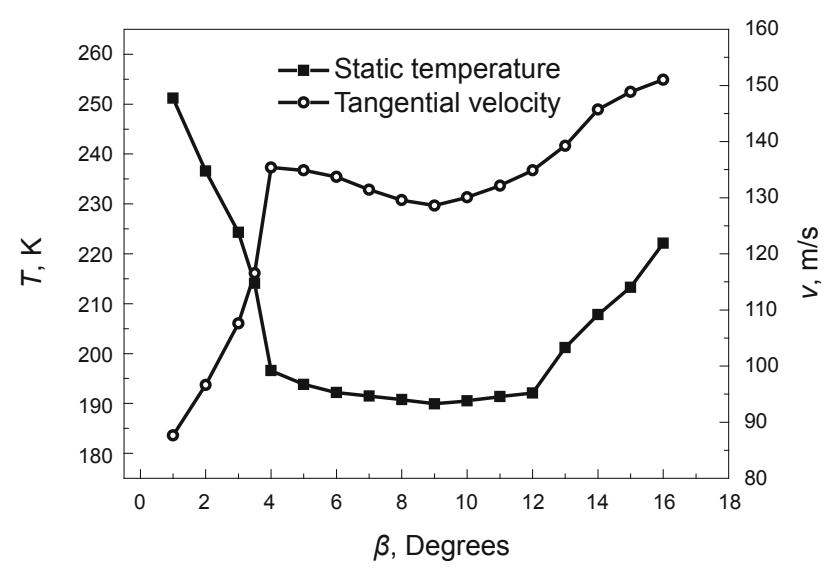

Fig. 10 Effects of divergent angle 
and the internal disturbance would be aggravated, which leads to an increase in the inner friction loss. As a result, the static temperature at the outlet of the nozzle increases. Therefore, the swirling separation performance decreases.

\section{Conclusions}

1) A new convergent-divergent nozzle was designed for the purpose of wet natural gas separation. The presence of the central body can ensure that the vortex is concentric, allowing the separation efficiency to be significantly improved.

2) In this new designed nozzle, gas expands to supersonic velocities, resulting in lower temperatures $\left(-80{ }^{\circ} \mathrm{C}\right)$. This causes the condensation and nucleation of water and heavy hydrocarbons. Meanwhile, swirls are formed when the fluid enters the swirling vanes located at the entrance of the nozzle, and the strength of vorticity is strongly increased due to the contraction of the central body. The very high centrifugal acceleration is in the order of 482,400 times the acceleration of gravity. Under the combined effect of the condensation and centrifugal field, water and heavy hydrocarbons can be condensed and separated from natural gas.

3) The main parameters of gas flow are non-uniformly distributed in the radial direction because of the special features of the geometry. The tangential velocity and the static temperature are uniformly distributed correspondingly in the radial central region of the channel, taking values from -80 to $-55^{\circ} \mathrm{C}$ and $220,000 \mathrm{~g}$ to $500,000 \mathrm{~g}$, respectively. However, the non-uniformity of radial distribution causes high gradients of gas dynamic parameters in the vicinity of the channel walls, which may impair the process of separation.

4) The geometry of the nozzle significantly affects the process of the natural gas separation. Good separation performance can be obtained with an increase in the nozzle convergent angle. When the divergent half-angle is kept at $2^{\circ}-6^{\circ}$, the swirling natural gas can be well separated. However, if the divergent half-angle is less than $2^{\circ}$ or more than $6^{\circ}$, the separation performance of the nozzle declines.

\section{Acknowledgements}

This study is supported by the National High Technology Research and Development Program of China ("863 program", No. 2007AA09Z301) and the National Major Science \& Technology Specific Projects (No. 2008ZX05017004).

\section{References}

Alferov V I, Baguirov L A, Feygin V I, et al. Method and apparatus for liquefying a gas. European Patent: EP1131588. February 25, 2004 Alfyorov V, Bagirov L, Dmitriev L, et al. Supersonic nozzle efficiently separates natural gas components. Oil \& Gas Journal. 2005. 5(103): 53-58

Betting M, Van Holten T and Van Veen J M. Nozzle for supersonic gas flow and an inertia separator. US Patent: 6524368. February 25, 2003

Betting M and Epsom H D. Supersonic separator gains market acceptance. World Oil. 2007. 228(4): 197-200

Boerner C J, Sparrow E M and Scott C J. Compressible swirling flow through convergent-divergent nozzles. Heat and Mass Transfer. 1972. 5(2): 101-115

Brouwer J M and Epsom H D. Twister supersonic gas conditioning for unmanned platforms and subsea gas processing. Paper SPE 83977 presented at the Proceedings of the Offshore Europe Conference, 2-5 September 2003, Aberdeen, United Kingdom

Cao X W, Chen L, Lin Z H, et al. The nozzle used in supersonic swirling separators. Natural Gas Industry. 2007. 27(7): 112-114 (in Chinese)

Jassim E, Abdi M A and Muzychka Y. Computational fluid dynamics study for flow of natural gas through high-pressure supersonic nozzles: Part 1. Real gas effects and shockwave. Petroleum Science and Technology. 2008a. 26(15): 1757-1772

Jassim E, Abdi M A and Muzychka Y. Computational fluid dynamics study for flow of natural gas through high-pressure supersonic nozzles: Part 2. Nozzle geometry and vorticity. Petroleum Science and Technology. 2008b. 26(15): 1773-1785

Jiang W M, Liu Z L, Liu H W, et al. Numerical simulation of a twocomponent mixture in one-dimension supersonic separator. Acta Petrolei Sinica (Petroleum Processing Section). 2008a. 24(6): 697701 (in Chinese)

Jiang W M, Liu Z L, Liu H W, et al. Two dimensional simulation and analysis of the flow in a supersonic separator. Journal of Engineering Thermophysics. 2008b. 29(12): 2119-2121 (in Chinese)

Karimi A and Abdi M A. Selective dehydration of high-pressure natural gas using supersonic nozzles. Chemical Engineering and Processing. 2009. 48(1): 560-568

Liu H W, Liu Z L, Feng Y X, et al. Characteristic of a supersonic swirling dehydration system of natural gas. Chinese Journal of Chemical Engineering. 2005. 13(1): 9-12

Man H C, Duan J and Yue T M. Design and characteristic analysis of supersonic nozzle for high pressure laser cutting. Journal of Materials Processing Technology. 1997. 63: 217-222

Okimoto F and Brouwer J M. Supersonic gas conditioning. World Oil. 2002. 223(8): 89-91

Patankar S V. Numerical Heat Transfer and Fluid Flow. New York: McGraw-Hill. 1980. 131-134

Patankar S V and Spalding D B. A calculation procedure for heat, mass and momentum transfer in three-dimensional parabolic flows. International Journal of Heat and Mass Transfer. 1972. 15(10): 17871806

Pope S B. Turbulent Flows. Cambridge: Cambridge University Press. 2000. 382-383

Wen C, Cao X W and Wu L H. Structure design and numerical simulation of a novel supersonic swirling separator. Journal of China University of Petroleum (Edition of Natural Science). 2010. 34(4): 119-122

(Edited by Sun Yanhua) 